\title{
The turbulent environment of low-mass dense cores
}

\author{
E. Falgarone ${ }^{1}$, \\ P. Hily-Blant ${ }^{2}$, J. Pety ${ }^{1,2}$ and G. Pineau des Forêts ${ }^{3,1}$ \\ ${ }^{1}$ LERMA/LRA, Ecole Normale Supérieure, \\ 24 rue Lhomond, 75005 Paris, France \\ email: edith@lra.ens.fr \\ ${ }^{2}$ IRAM, 300 rue de la Piscine, 38406 Saint Martin d'Hères, France \\ email: hilyblan@iram.fr, pety@iram.fr \\ ${ }^{3}$ IAS, Université Paris-Sud, 91405 Orsay, France \\ email:guillaume.pineaudesforets@ias.u-psud.fr
}

\begin{abstract}
The signatures of intermittent dissipation of turbulent energy have been sought in the translucent environment of a low-mass dense core. Molecular line observations reveal a network of narrow filamentary structures, found on statistical grounds to be the locus of the largest velocity shears. Three independent properties of these structures make them the plausible sites of intermittent dissipation of turbulence: (1) gas there is warmer and more diluted than average, (2) it bears the signatures of a non-equilibrium chemistry triggered by impulsive heating due to turbulence dissipation, and (3) the power that these structures radiate in the gas cooling lines (mostly $\mathrm{H}_{2}$ ) is so large that it balances the total energy injection rate of the turbulent cascade, for a volume filling factor of only a few percents, consistent with other observations in the Solar Neighborhood. These filamentary structures may act as tiny seeds of gas condensation in diffuse molecular gas. They do not exhibit the properties of steady-state low-velocity magnetohydrodynamic (MHD) shocks, as presently modelled.
\end{abstract}

Keywords. Turbulence, astrochemistry, MHD, ISM:evolution, ISM:structure, ISM:molecules, ISM:cloud, stars:formation, lines:profiles, radio lines:ISM

\section{Introduction}

Star formation has at least two (presumably coupled) prerequisites: the growth of a dense core up to a gravitationally unstable state and dissipation of a large fraction of the gas turbulent specific energy (see the detailed reviews of Elmegreen \& Scalo 2004 and MacLow \& Klessen 2004). The timescales over which these processes occur are critical inputs in the estimates of the star formation efficiency. Yet, little is known on the steps at the origin of the formation of dense cores, nor on those causing turbulence dissipation of interstellar turbulence. Whether it occurs in shocks or in intense velocity shears, turbulent dissipation is intermittent in space and time i.e. concentrated in a small fraction of the volume (Kolmogorov 1962, Landau \& Lifchitz 1959). Therefore, a scale may exist where, locally, the heating rate due to turbulence dissipation dominates all other heating mechanisms, and induces specific signatures in the gas.

We have sought such signatures in maps of molecular lines in the environment of lowmass dense cores still in the early stages of their evolution. Intermittency manifests itself in the non-Gaussian tails of the probability distribution functions of velocity increments (e.g. Frisch et al. 1978, Porter et al. 1994, Pety \& Falgarone 2000). We followed the statistical method of Lis et al. (1996), who showed that the increments of line centroid velocities (CVIs) measured between two different line of sight (LOS) trace a quantity 


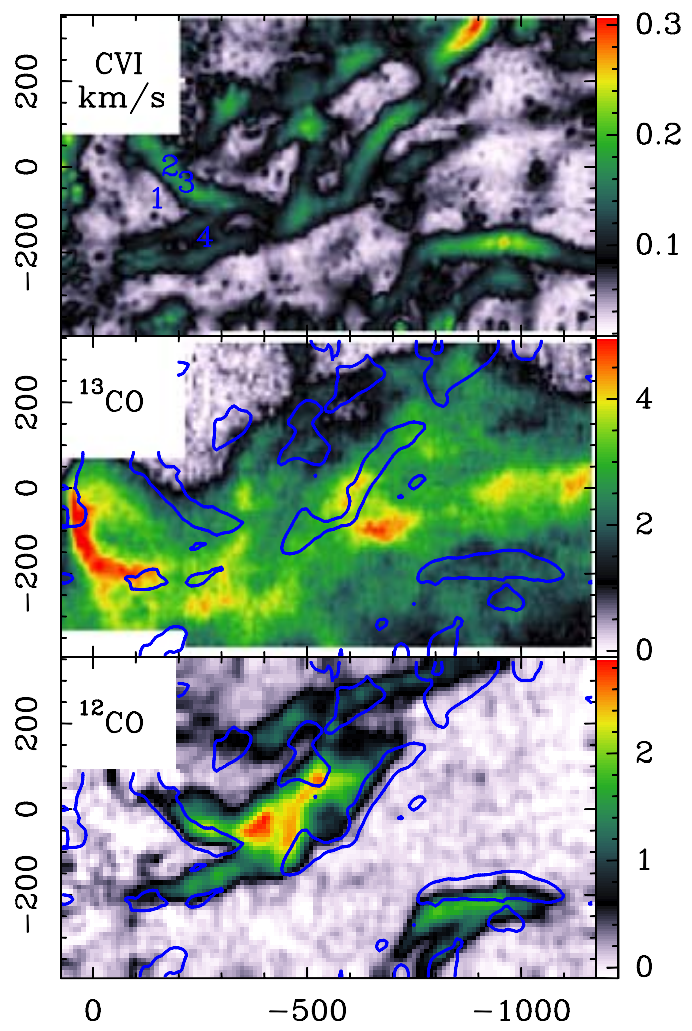

Figure 1. CO observations of the parsec scale environment of a dense core (IRAM-30m observations, resolution 22 arc sec or $\sim 0.02 \mathrm{pc}$ ). Top: map of the largest centroid velocity increments (or velocity shears) for lags of 3 pixels expressed in $\mathrm{km} \mathrm{s}^{-1}$ (Hily-Blant 2004, Hily-Blant et al., in prep.). The 4 blue numbers give the positions observed in $\mathrm{HCO}^{+}(1-0)$. Middle: The most opaque regions traced by the ${ }^{13} \mathrm{CO}(1-0)$ line integrated intensity (greyscale in $\mathrm{K} \mathrm{km} \mathrm{s}^{-1}$ ) and those of largest velocity shears (blue contours from top panel). The dense core is visible at the eastern edge of the map. Bottom: The gas optically thin in ${ }^{12} \mathrm{CO}(1-0)$, thus warmer and more diluted than average (greyscale in $\mathrm{K} \mathrm{km} \mathrm{s}^{-1}$ ) and the regions of largest velocity shears (blue contours) (Hily-Blant \& Falgarone 2006). The offsets are in arcsec.

related to the LOS average of the plane-of-the-sky projection of the vorticity. It is thus possible, from the CVIs statistics computed in a map of spectra, to approach that of the velocity shears and, most importantly, find the subset of space where the departures from a Gaussian distribution occur.

\section{Small-scale velocity structures in a dense core environment}

An example is shown in Fig. 1 that displays the parsec scale environment of a lowmass dense core (Heithausen et al. 2002) mapped with the IRAM-30m telescope in the ${ }^{12} \mathrm{CO}$ and ${ }^{13} \mathrm{CO} J=2-1$ and 1-0 transitions (Hily-Blant 2004). The dense core, visible in the East of the central panel, is not isolated but is connected, in space and velocity, to a parsec-scale tail of $\sim 2 M_{\odot}$, comparable to its own mass. Longitudinal velocity gradients of a few $\mathrm{km} \mathrm{s}^{-1} \mathrm{pc}^{-1}$ may be interpreted as slow infalling motions onto the core. The upper panel of Fig. 1 shows a new kind of small-scale structures: those traced by the extrema of CVIs, i.e. extrema of velocity shear (Pety \& Falgarone 2003, Hily-Blant et al., in prep.). They form a conspicuous network of filamentary structures of thickness 


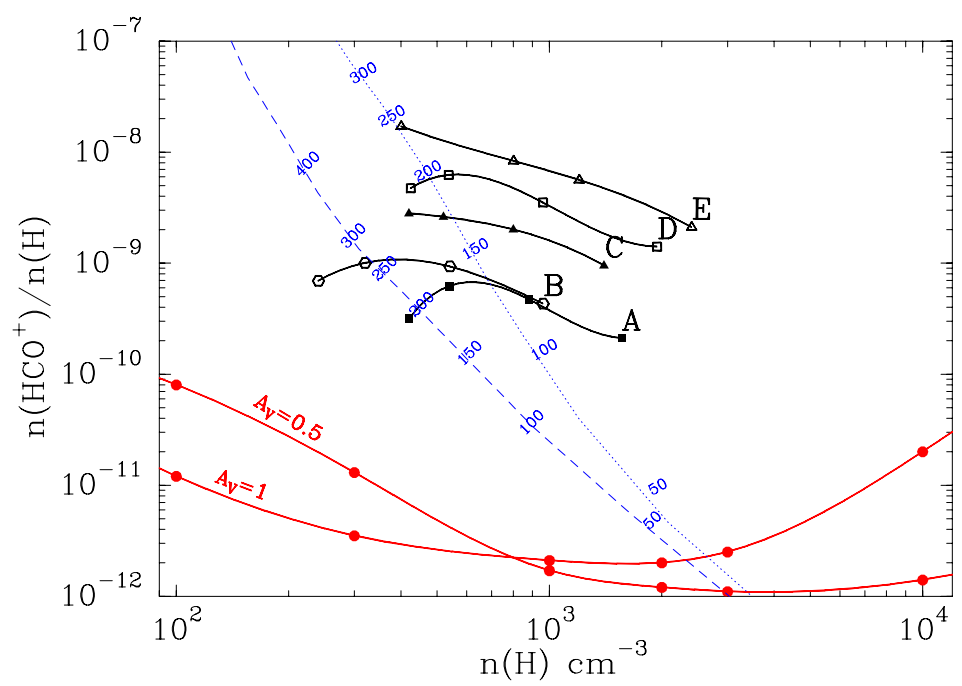

Figure 2. Domain of $\mathrm{HCO}^{+}$abundances derived from observed spectra (black curves): the $\mathrm{HCO}^{+}$line intensity increases from curve A to E. The kinetic temperature of the solutions increases from right to left on each curve, and the symbols are located at $T_{k}=20,40,100$ and $200 \mathrm{~K}$. Predictions of steady-state chemistry computed for two different UV shieldings, bracketing that of the core environment (red curves). Time-dependent $\mathrm{HCO}^{+}$abundances along two isobaric cooling sequences of gas previously enriched in a burst of dissipation of turbulence (same initial density and two different UV shieldings, $A_{v}=0.5$ and $1 \mathrm{mag}$, blue curves). Labels are gas temperature dropping with time. Models meet observations in the range $T=100-200 \mathrm{~K}$, $n_{\mathrm{H}}=200-10^{3} \mathrm{~cm}^{-3}$ (from Falgarone et al. 2006).

$\sim 0.05 \mathrm{pc}$, unrelated to the tail of dense gas traced by ${ }^{13} \mathrm{CO}$ emission (central panel) and instead clearly associated with gas optically thin in ${ }^{12} \mathrm{CO}(1-0)$ (bottom panel), shown to be warmer $\left(T_{k}>30 \mathrm{~K}\right)$ and more diluted $\left(n_{\mathrm{H}_{2}}<10^{3} \mathrm{~cm}^{-3}\right)$ than the average gas in the core environment (Hily-Blant \& Falgarone 2006). Their preferential orientation is close to the direction of the magnetic fields measured one degree away (Heiles 2000).

Observations of one of these filamentary structures with the IRAM Plateau de Bure Interferometer $(\mathrm{PdBI})$ in the ${ }^{12} \mathrm{CO}(1-0)$ line reveal still thinner structures of thickness $\sim 800 \mathrm{AU}$, separated by less than 10 arsec (or $7.5 \mathrm{mpc}$ ) in projection and $3.5 \mathrm{~km} \mathrm{~s}^{-1}$ in velocity, all the emission at intermediate velocities being resolved out. Such a pattern may be viewed as a large local velocity shear of $\sim 200 \mathrm{~km} \mathrm{~s}^{-1} \mathrm{pc}^{-1}$, or in turn as an impulsive event of timescale $\tau \sim 3000$ yr (Falgarone et al., in prep.).

\section{Non-equilibrium chemistry in regions of largest velocity-shear}

If large amounts of suprathermal energy are deposited in small regions over such short timescales, the thermal and chemical evolution of the gas there should be different from that of the rest of the volume. We have sought such differences by observing the four positions shown in Fig. 1 in the $\mathrm{HCO}^{+}(1-0)$ line. These four positions sample the whole range of velocity-shears in the map from a maximum (\#2 and 3) to a minimum (\#1).

The observed $\mathrm{HCO}^{+}$abundances are similar to those obtained by Liszt \& Lucas (1996) and Lucas \& Liszt (2000) in absorption against extragalactic continuum sources, and exceed by more than one order of magnitude the predictions of steady-state chemistry in gas primarily heated by UV photons and cosmic rays (Fig. 2). In contrast, they can be understood in the framework of the non-equilibrium model described below (Falgarone et al. 2006). 

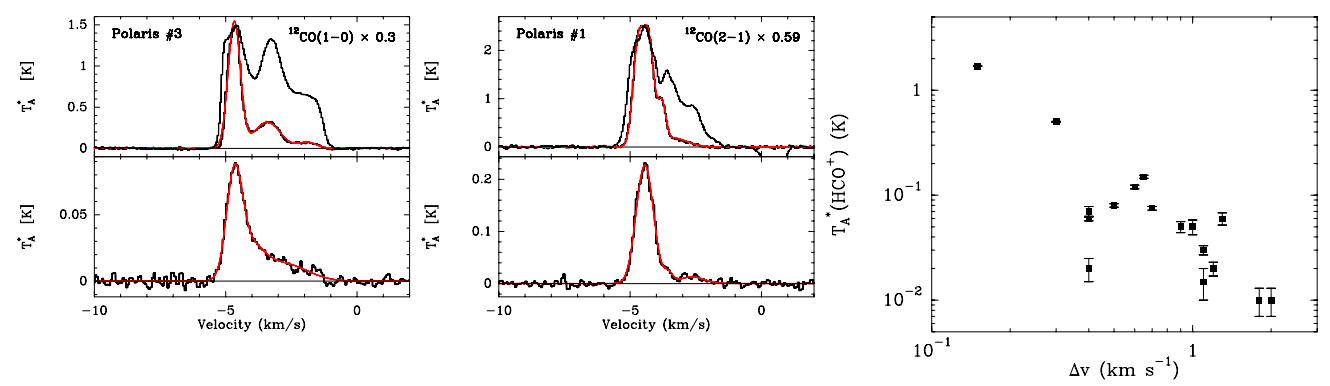

Figure 3. Left: ${ }^{12} \mathrm{CO}$ and ${ }^{13} \mathrm{CO}(1-0)$ (top) and $\mathrm{HCO}^{+}(1-0)$ (bottom) spectra at positions \#3 and \#1. Right: $\mathrm{HCO}^{+}$line temperature versus velocity width of all the components identified in the $\mathrm{HCO}^{+}$line profiles (4 positions). The observed scaling, close to $T_{L} \propto \Delta v^{-2}$, means $N\left(\mathrm{HCO}^{+}\right) \approx \Delta v^{-1}$ since the lines are almost optically thin.

It is out of reach of present computational capabilities to follow the non-equilibrium chemistry that develops in a turbulent medium at time and size scales close to those of dissipation. An hybrid approach was adopted by Joulain et al. (1998): the time-dependent Lagrangian evolution of a cell of gas is computed as it spirals into a steady-state magnetized Burgers vortex, chosen as a template of turbulent dissipative structure (Moffatt, Kida \& Ohkitani 1994). Because of the short timescales, neutrals are only partially coupled to the ions and field. Intense dissipation occurs, both in the layers of largest velocity shear and those of large ion-neutral drift. As the gas cell escapes these active layers, it cools down. The temperature excursion therefore includes (1) an impulsive heating over a few $100 \mathrm{yr}$ from $T_{k}=80 \mathrm{~K}$ to about $10^{3} \mathrm{~K}$, followed by (2) an isobaric relaxation that lasts up to $10^{4} \mathrm{yr}$ (Falgarone et al. 2006).

The main interest of such an hybrid model is to show (1) that chemistry swiftly reacts to sharp temperature variations, triggering a warm chemistry by activating a set of endoenergetic reactions that enhance the local production of molecules like $\mathrm{CH}^{+}, \mathrm{HCO}^{+}$, $\mathrm{CH}, \mathrm{OH}$ and $\mathrm{H}_{2} \mathrm{O}$ by several orders of magnitude, and (2) that molecules formed there survive for more than a thousand years after the gas has escaped the active layers, while radiative cooling drives the condensation of the gas. Interestingly, Fig. 2 shows that such non-equilibrium models meet the observational constraints in the range $T=100-200$ $\mathrm{K}, n_{\mathrm{H}}=200-10^{3} \mathrm{~cm}^{-3}$. Thus, alike MHD shocks, intense and coherent velocity shears induce thermal and chemical excursions in the diffuse gas which eventually turn into high density structures at small scale.

The fact that impulsive heating generates sharp gradients of temperature and molecular abundance over scales much smaller than that of the beam resolution (here, about $0.02 \mathrm{pc}$ ) is supported by the following. The four observed $\mathrm{HCO}^{+}$spectra have multiple velocity components of different linewidths, the broadest present only at positions \# 2 and 3 (see Fig. 3). The $\mathrm{HCO}^{+}$line intensity of these components increases steeply as their linewidth decreases (Fig. 3, right). Since the lines are close to be optically thin, the column density of $\mathrm{HCO}^{+}$also increases as the linewidth decreases, as $N\left(\mathrm{HCO}^{+}\right) \approx \Delta v^{-1}$. This suggests that: (1) the observed chemical enrichment proceeds at the expense of turbulent energy, traced by the linewidth of each component and (2) that in each beam, gas being enriched, still in active layers (the broad and weak components) coexists with chemically enriched gas, already relaxing (the intense and narrower components). As expected, the former is almost absent from the spectrum observed at position $\# 1$, an inactive location, and most prominent at positions \#2 and 3, located on an extremum of velocity-shear (Fig. 3). 


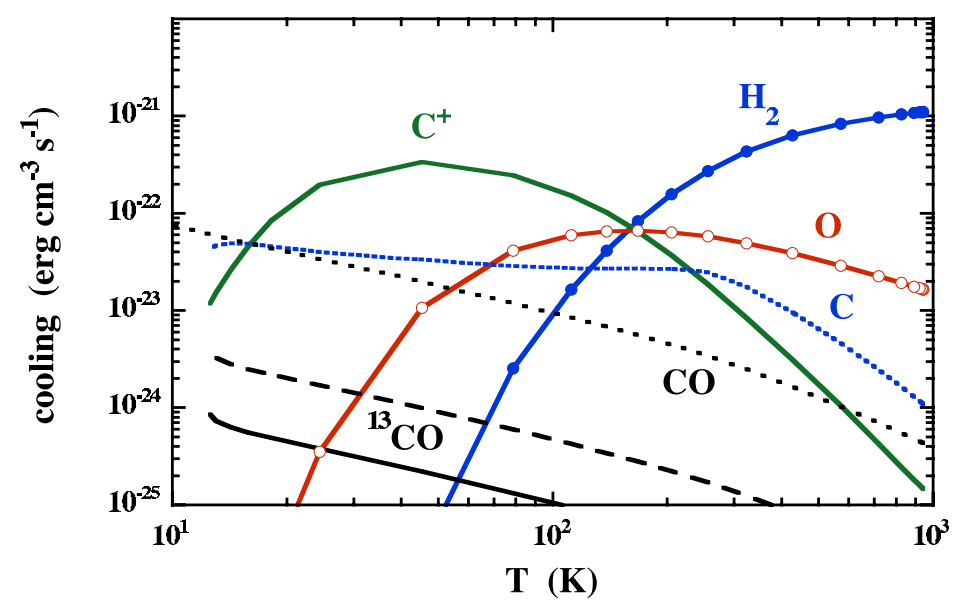

Figure 4. Radiative cooling curves of the gas along an isobaric thermal and chemical relaxation. The time-dependent evolution starts at $T_{k}=10^{3} \mathrm{~K} . \mathrm{H}_{2}$ line emission dominates the cooling at high temperatures, and CO lines are never important unless the gas is cold (optically thin (dotted) and thick (dashed) cases).

Alternative scenarios have been proposed to explain the large abundances of $\mathrm{CH}^{+}$, $\mathrm{HCO}^{+}, \mathrm{OH}$ and $\mathrm{H}_{2} \mathrm{O}$ in the diffuse ISM. They invoke chemistry developing in warm interfaces between diffuse clouds and the warm intercloud medium (Nguyen, Hartquist \& Williams 2001, Lesaffre, Gerin, Hennebelle 2006) or in myriads of low-velocity MHD shocks (e.g. Flower \& Pineau des Forêts 1998, Gredel et al. 2002).

\section{Energetics}

The radiative cooling of the gas formerly heated by a dissipation burst and chemically enriched drives its condensation during the relaxation phase. The contribution of all the species contributing is displayed in Fig. 4 as a function of temperature (or time). The pure rotational lines of $\mathrm{H}_{2}$ dominate the cooling in the warmest stages. At the opposite, between $T_{k}=200$ and $100 \mathrm{~K}$, the temperature range of the solutions, the cooling is shared almost equally between $\mathrm{H}_{2}$ and the fine structure lines of $\mathrm{C}^{+}, \mathrm{O}$ I and $\mathrm{C}$ I. Note that in that temperature range, $\mathrm{CO}$ lines have a negligible contribution since $\Lambda_{\text {tot }}=\Lambda_{\mathrm{H}_{2}}+\Lambda_{\mathrm{C}^{+}}+\Lambda_{\mathrm{OI}}+\Lambda_{\mathrm{CI}}+\Lambda_{\mathrm{CO}}=30$ to $40 \Lambda_{\mathrm{CO}}$.

The radiative cooling rate of the gas in its relaxation phase is much larger than the average turbulent heating rate in this dense core environment, estimated to be $\bar{\epsilon}_{\text {turb }}=$ $6 \times 10^{-24} \mathrm{erg} \mathrm{cm}^{-3} \mathrm{~s}^{-1}$ (Hily-Blant \& Falgarone 2006). For $T_{k}=100-200 \mathrm{~K}, \Lambda_{\text {tot }}=$ $2-3 \times 10^{-22}$ erg cm ${ }^{-3} \mathrm{~s}^{-1}$ (Fig. 4). So, even a tiny fraction of warm gas (transient but permanently replenished by dissipation) is able to radiate away the turbulent energy input. The volume filling factor required for the balance is as small as: $f_{v}=\bar{\epsilon}_{t u r b} / \Lambda_{t o t}=$ 0.02 to 0.03 . Only a few percent of warm gas in the case of the field under study, are sufficient to radiate the input of energy of the turbulent cascade.

It is all the more interesting that this fraction is also that required, in the Solar Neighborhood, to reproduce the observed abundances of $\mathrm{CH}^{+}$(Crane, Lambert \& Sheffer 1995, Gredel 1997) and $\mathrm{H}_{2} \mathrm{O}$ found by the $S W A S$ satellite in diffuse molecular clouds (Neufeld et al. 2002, Plume et al. 2004). Similar findings are inferred from ISO-SWS observations of pure rotational lines of $\mathrm{H}_{2}$ across 15 magnitudes of mostly diffuse gas in the Galaxy (Falgarone et al. 2005). The warm $\mathrm{H}_{2}$ is distributed in a large number of either low velocity MHD shocks or small scale intense velocity-shears, occupying only a 
few percent of the line of sight. The same fraction of warm $\mathrm{H}_{2}$ that cannot be heated by the sole UV photons is also derived from far UV spectroscopy with FUSE (Gry et al. 2002, Lacour et al. 2005). Warm glitters seem to be ubiquitous, and presumably trace the intermittent dissipation of suprathermal turbulence in the cold gas.

\section{Summary and open questions}

Signatures of intermittent dissipation of turbulent energy may have been detected for the first time: they appear as extrema of velocity-shears, forming a network of warm and narrow filamentary structures, in the environment of a dense core. These structures are the sites of non-equilibrium chemistry proceeding at the expense of the turbulent energy. Their radiative cooling is powerful and they balance the average turbulent heating while filling only a few percent of the volume. The actual scale at which dissipation of turbulence takes place has not be determined yet. It may be smaller than $800 \mathrm{AU}$ and accessible only to the ALMA interferometer. The full nature of the dissipation process is not understood either: the observational constraints (small sizescale, energy release sufficient to drive the warm chemistry, lack of dense post-shock gas) make the observed structures inconsistent with steady-state low-velocity MHD shocks, as presently modelled.

\section{References}

Crane P., Lambert D.L. \& Sheffer Y. 1995, ApJS 99, 107

Elmegreen B.G. \& Scalo J. 2004, ARAA 42, 211

Falgarone E., Pineau des Forêts G., Hily-Blant P. \& Schilke P. 2006, A\&A 4 452, 511

Falgarone E., Verstraete L., Pineau des Forêts G. \& Hily-Blant P. 2005, A $\mathscr{S} A$ 433, 997

Falgarone, E., Pety J. \& Hily-Blant, P., in preparation

Flower D. \& Pineau des Forêts G. 1998, MNRAS 297, 1182

Frisch U., Sulem P.-L. \& Nelkin M. 1978, JFM 87, 719

Gredel R. 1997, A\&A 320, 929

Gredel R., Pineau des Forêts G. \& Federman S.R. 2002, A\&3A 389, 993

Gry, C., Boulanger, F. Nehmé, C., et al. 2002, A\& A 391, 675

Heiles C. 2000, AJ 119, 923

Heithausen A., Bertoldi F. \& Bensch F. 2002, A\&A 383, 591

Hily-Blant, P. 2004, PhD, Université Paris-Sud

Hily-Blant P. \& Falgarone E. 2006, A\&A accepted

Hily-Blant P., Pety J. \& Falgarone E., in preparation

Joulain K., Falgarone E., Pineau des Forêts G. \& Flower D. 1998, A $\& A$ 340, 241

Kolmogorov, A.N. 1962, JFM 13, 82

Lacour S., Ziskin V., Hébrard G., et al. 2005, ApJ 627, 251

Lambert D.L. \& Danks A.C. 1986, ApJ 303, 401

Landau L.D. \& Lifchitz E.M. 1959, Fluid Mechanics, Addison-Wesley

Lesaffre P., Gerin M. \& Hennebelle P. 2006, A\& $A$ submitted

Lis D., Pety J., Phillips T.G. \& Falgarone E. 1996, ApJ 463, 623

Liszt H.S. \& Lucas R. 1996, A\&GA 307, 237

Lucas R. \& Liszt H.S. 2000, A $\& A 355,333$

MacLow M.-M. \& Klessen R. 2004, Rev.Mod.Phys. 76, 125

Moffatt H.K., Kida S. \& Ohkitani K. 1994, JFM 259, 241

Neufeld D.A., Kaufman M.J., Goldsmith P.F., et al. 2002, ApJ 580, 278

Nguyen, T. K., Hartquist, T. W. \& Williams, D. A. 2001, A\& $A$ 366, 662

Pety, J. \& Falgarone E. 2000, A\&A 356, 279

Pety, J. \& Falgarone E. 2003, A\&SA 412, 417

Plume R., Kaufman M.J., Neufeld D.A. et al. 2004, ApJ 605, 247

Porter D.H., Pouquet A. \& Woodward P.R. 1993, Phys. Fluids 6, 2133 


\section{Discussion}

MAC Low: Why can't these thin, impulsively heated objects be small shocks in a dissipating supersonic turbulent flow?

FALGarone: Good question! We have no observational evidence for any condensed postshock layer. But it may well be that the column density of this thin dense layer is still too low to be detected, even with the Plateau de Bure interferometer. If this is true, models will have to explain such thin post-shock layers in the presence of magnetic fields presumably parallel to the shock. 